\title{
Guiding mesenchymal stem cell differentiation using mesoporous silica nanoparticle-based films
}

Citation for published version (APA):

Andree, L., Barata, D., Sutthavas, P., Habibovic, P., \& van Rijt, S. (2019). Guiding mesenchymal stem cell differentiation using mesoporous silica nanoparticle-based films. Acta Biomaterialia, 96, 557-567. https://doi.org/10.1016/j.actbio.2019.07.008

Document status and date:

Published: 15/09/2019

DOI:

10.1016/j.actbio.2019.07.008

Document Version:

Publisher's PDF, also known as Version of record

Document license:

Taverne

\section{Please check the document version of this publication:}

- A submitted manuscript is the version of the article upon submission and before peer-review. There can be important differences between the submitted version and the official published version of record.

People interested in the research are advised to contact the author for the final version of the publication, or visit the DOI to the publisher's website.

- The final author version and the galley proof are versions of the publication after peer review.

- The final published version features the final layout of the paper including the volume, issue and page numbers.

Link to publication

\footnotetext{
General rights rights.

- You may freely distribute the URL identifying the publication in the public portal. please follow below link for the End User Agreement:

www.umlib.nl/taverne-license

Take down policy

If you believe that this document breaches copyright please contact us at:

repository@maastrichtuniversity.nl

providing details and we will investigate your claim.
}

Copyright and moral rights for the publications made accessible in the public portal are retained by the authors and/or other copyright owners and it is a condition of accessing publications that users recognise and abide by the legal requirements associated with these

- Users may download and print one copy of any publication from the public portal for the purpose of private study or research.

- You may not further distribute the material or use it for any profit-making activity or commercial gain

If the publication is distributed under the terms of Article $25 \mathrm{fa}$ of the Dutch Copyright Act, indicated by the "Taverne" license above, 
Full length article

\title{
Guiding mesenchymal stem cell differentiation using mesoporous silica nanoparticle-based films
}

\author{
Lea Andrée, David Barata, Pichaporn Sutthavas, Pamela Habibovic, Sabine van Rijt* \\ Department of Instructive Biomaterials Engineering, MERLN Institute for Technology-Inspired Regenerative Medicine, Maastricht University, P.O. Box 616, 6200 MD Maastricht, \\ the Netherlands
}

\section{A R T I C L E I N F O}

\section{Article history:}

Received 20 March 2019

Received in revised form 2 July 2019

Accepted 3 July 2019

Available online 5 July 2019

\section{Keywords:}

Mesoporous silica nanoparticles

Drug delivery

Nanoparticle film

Supported lipid bilayers

Guided differentiation

\begin{abstract}
A B S T R A C T
The development of smart interfaces that can guide tissue formation is of great importance in the field of regenerative medicine. Nanoparticles represent an interesting class of materials that can be used to enhance regenerative treatments by enabling close control over surface properties and directing cellular responses. Moreover, nanoparticles can be used to provide temporally controlled delivery of (multiple) biochemical compounds. Here, we exploited the cargo loading and surface functionalization properties of mesoporous silica nanoparticles (MSNs) to design films that can guide human mesenchymal stem cell (hMSC) differentiation towards the osteogenic lineage. We developed biocompatible MSN-based films that support stem cell adhesion and proliferation and demonstrated that these MSN films simultaneously allowed efficient local delivery of biomolecules without effecting film integrity. Films loaded with the osteogenesis-stimulating drug dexamethasone (Dex) were able to induce osteogenic differentiation of hMSCs in vitro. Dex delivery from the films led to increased alkaline phosphatase levels and matrix mineralization compared to directly supplementing Dex to the medium. Furthermore, we demonstrated that Dex release kinetics can be modulated using surface modifications with supported lipid bilayers. Together, these data demonstrate that MSN films represent an interesting approach to create biomaterial interfaces with controllable biomolecule release and surface properties to improve the bioactivity of biomaterials.
\end{abstract}

\section{Statement of significance}

Engineering surfaces that can control cell and tissue responses is one of the major challenges in biomaterials-based regenerative therapies. Here, we demonstrate the potential of mesoporous silica nanoparticles (MSNs) as drug-delivering surface coatings. First, we show differentiation of mesenchymal stem cells towards the bone lineage when in contact with MSN films loaded with dexamethasone. Furthermore, we demonstrate that modification of MSNs with supported lipid bilayer allows control over drug release dynamics and cell shape. Given the range of loadable cargos and the tunability of release kinetics, MSN coatings can be used to mimic the sequential appearance of bioactive factors during tissue regeneration, which will ultimately lead to biomaterials with improved bioactivity.

(c) 2019 Acta Materialia Inc. Published by Elsevier Ltd. All rights reserved.

\section{Introduction}

Currently, a major focus in the field of regenerative medicine is the engineering and development of biomaterials that can repair or regenerate damaged cells, organs and tissues. Such materials are expected to temporarily provide sufficient (mechanical) support, while serving as a matrix for new tissue formation by which they are eventually replaced. To effectively direct tissue regeneration,

\footnotetext{
* Corresponding author.

E-mail address: s.vanrijt@maastrichtuniversity.nl (S. van Rijt).
}

control over key stem cell fate processes such as adhesion, proliferation, migration, and differentiation is needed. Within the body, the extracellular matrix (ECM) provides multiple chemical and biophysical cues that can be sensed by cells to direct these processes. Synthetic approaches that mimic these cues have been an important strategy in the development of instructive biomaterials for tissue engineering and other regenerative medicine applications. For example, tuning material properties, such as surface chemistry and surface topography, can guide stem cells to align or even differentiate [1-4]. Chemical approaches include incorporation in, and release of biomolecules (e.g., growth factors) from biomaterial 
carriers. A well-known example is the release of bone morphogenetic protein 2 (BMP-2) to stimulate bone growth [5-7], which has obtained market approval. However, biomolecule release from these scaffolds needs to be tightly controlled to avoid initial burst release, potentially causing serious side effects as a result of local supraphysiological concentrations. In addition, uncontrolled biomolecule release minimizes the efficacy as prolonged chemical signalling is not possible [6-8]. Next to other approaches using polymers, and microparticles [9-11], nanoparticle-based carriers could increase control over the pharmacokinetics of such agents owing to their ability to carry high concentrations of (insoluble) biomolecules, while protecting them from degradation. In addition, nanoparticles can be surface-modified to allow for sustained drug delivery to improve the biological performance of biomaterials by providing temporally controlled release over (multiple) biochemical cues.

Next to their drug delivery capabilities, nanoparticles are interesting materials for designing biomaterial interfaces by offering ways to closely control surface topographical and chemical properties to direct adhesion, differentiation and integration of (stem) cells into the surrounding environment. In this context, mesoporous silica nanoparticles (MSNs) show interesting properties for regenerative medicine as they are biocompatible and have shown promise in several biomedical fields such as in cancer therapy and tumour imaging $[12,13]$. In addition, the inherent material properties of MSNs allow for the development of mechanically stronger materials with higher bioactivity. For example, the incorporation of silica nanoparticles into hydrogels has resulted in increased compressive strength and fracture toughness by an order of magnitude compared to non-fortified hydrogels [14-16]. Several studies reported that the incorporation of MSNs in biomaterials can improve their biological performance, such as enhanced initial cell adhesion, cell viability, cell spreading, and metabolic activity of stem cells [17-19]. Moreover, MSNs are easy to functionalize and their surface chemistry can be used to modulate cell-material interactions. Latest advances in the synthesis of MSNs allow capping or closing the pore entry using polymers or lipids, thereby enabling better control over cargo release [20-22]. Promising results on the use of surface modifications to control drug release have been obtained by using supported lipid bilayers (SLB) to prevent premature cargo release from MSNs [23]. Furthermore, SLBs allow control over the cell-material interface by the ability to change the chemistry (i.e., lipid content), fluidity (i.e., lipid phase) and incorporation of additional ligands (e.g., adhesion peptides) [24].

Here, we hypothesized that the cargo loading and favourable surface properties of MSNs can be leveraged to design films that can guide human mesenchymal stem cell (hMSC) behaviour. The aim was to form biocompatible, stable MSN-based films that support stem cell adhesion and proliferation. The MSNs porous structure was used for delivering dexamethasone (Dex) as a showcase, which is a known stimulator of osteogenic differentiation. The ability of the films to induce the osteogenic differentiation of hMSCs and support their ECM formation in vitro was evaluated. Furthermore, we investigated the use of lipid surface modifications to modulate cell shape and to control release kinetics of incorporated cargo.

\section{Material and methods}

\subsection{Materials}

Tetraethyl orthosilicate (TEOS), 3-mercaptopropyl triethylsilane (MPTES), triethanolamine (TEA), 3-Aminopropyl triethoxysilane (APTES), cetyltrimethylammonium chloride (CTAC), ammonium fluoride, hydrochloric acid (37\%), ammonium nitrate, N,N-Dimethylformamide (DMF), ATTO 633-malemeide, 5/6carboxyfluorescein succinimidyl ester (NHS-FITC), phosphatebuffered saline (PBS), foetal bovine serum, ascorbic acid, bis[N,N-bis(carboxymethyl)aminomethyl]fluorescein (calcein), dexamethasone (Dex), $\beta$-glyerolphosphate (BGP) and hexamethyldisilazane (HMDS) were purchased from Sigma Aldrich $\mathrm{GmbH}$ (Germany). Absolute ethanol, paraformaldehyde (PFA), Triton $\mathrm{X}-100$, bovine serum albumin (BSA), Tween-20 and Alizarin Red $\mathrm{S}$ (sodium alizarin sulphonate) were purchased from VWR (US). Calcein AM blue, minimum essential medium alpha ( $\alpha M E M)$, L-glutamine and trypsin were purchased from Fisher Scientific (The Netherlands). Penicillin and streptomycin were purchased from Gibco Life Technologies (US). 1,2-dioleoyl-sn-glycero-3phosphocholine (DOPC) were purchased from Avanti Polar Lipids (US).

\subsection{MSN and MSN-DOPC synthesis}

Synthesis of MSNs with thiol core functionalization and amine surface functionalization was performed based on a sol-gel process reported elsewhere [25]. Synthesis of MSN-DOPC was performed as reported previously [26], using 1,2-dioleoyl-sn-glycero-3phosphocholine (DOPC) lipids. Further details on MSN synthesis and characterization can be found in the supporting information.

\subsubsection{Preparation of MSN and MSN-DOPC films}

MSN films were prepared using spin-coating on glass cover slips (diameter $22 \mathrm{~mm}$ ). Further details on film preparation and characterization can be found in the supporting information.

\section{3. hMSC cell culture}

hMSCs, obtained with full written consent from the donor, were expanded in $\alpha \mathrm{MEM}$ with addition of 10\% FBS, $2 \mathrm{mM}$ L-glutamine, $0.2 \mathrm{mM}$ ascorbic acid, $100 \mathrm{U} / \mathrm{ml}$ penicillin and $100 \mathrm{mg} / \mathrm{ml}$ streptomycin at $37{ }^{\circ} \mathrm{C}, 5 \% \mathrm{CO}_{2}$ in a humidified atmosphere. Cells between passage 4 and 6 were used for experiments. Seeding density varied depending on experimental aim (details on seeding densities can be found in individual experimental descriptions below). For cell seeding, $250 \mu$ l of cell suspension was carefully pipetted on the MSN and MSN-DOPC films, or uncoated glass cover slips, and cells were left to adhere for 5-6 $\mathrm{h}$ before addition of $2 \mathrm{ml}$ medium. For flow cytometry, cells were washed with PBS and detached from the cover slips using 0.05\% trypsin in EDTA. Detached cells were dispersed in cell culture medium and collected by centrifugation at $300 \mathrm{rcf}$ for $3 \mathrm{~min}$. The cell pellet was washed once with PBS and dispersed in $200 \mu$ PBS. Samples were stored on ice until analysis. To conserve cells for morphological analysis, cell monolayers were fixed at desired confluency using 4\% PFA in PBS. Samples were stored in PBS at $4{ }^{\circ} \mathrm{C}$ until staining.

\subsubsection{Assessment of cytotoxicity of MSN and MSN-DOPC films}

Cytotoxicity of MSN and MSN-DOPC films was evaluated by measuring extracellular lactate dehydrogenase (LDH) activity. hMSCs expanded in basic medium were detached at a confluence of $70-80 \%$ and seeded on MSN and MSN-DOPC films at a density of 5000 cells $/ \mathrm{cm}^{2}$. After 1,5 and 7 days of culture, aliquots of the medium were taken and the same volume of fresh medium was added to the wells. Cells grown on uncoated, $\mathrm{O}_{2}$ plasma surfaceactivated cover slips were used as negative control. To determine maximal extracellular LDH, cells grown on uncoated, $\mathrm{O}_{2}$ plasma surface-activated cover slips were permeabilized by adding $3 \%$ Triton $\mathrm{X}-100$ to the medium and incubating for $20 \mathrm{~min}$ at $37^{\circ} \mathrm{C}$ before taking aliquots of the medium. Amount of extracellular LDH was 
measured with PierceTM LDH Cytotoxicity Assay Kit (Thermo Fisher Scientific, US).

\subsubsection{Cellular adhesion on MSN films}

Cellular adhesion on MSN films was made visible by immunohistochemical staining of the focal adhesion protein vinculin. hMSCs were seeded on MSN and MSN-DOPC films (over the whole well surface) at a density of 1000 cells $/ \mathrm{cm}^{2}$ and 10,000 cells $/ \mathrm{cm}^{2}$ for culturing for 2 and 5 days, respectively. At 2 and $5 \mathrm{~d}$, cells were fixed with 4\% PFA. Prior to staining, cells were washed once with PBS and permeabilized with Triton X-100 (0.01\% (vol/vol) in PBS) for $10 \mathrm{~min}$ at room temperature followed by washing three times with PBS. Samples were then incubated for $60 \mathrm{~min}$ in blocking buffer (10\% BSA and $0.05 \%$ (vol/vol) Tween-20 in PBS) at room temperature. Mouse monoclonal IgG1 anti-vinculin antibody (SPM227, 1:500; Abcam) was used as the primary antibody and incubated with the cells overnight at $4{ }^{\circ} \mathrm{C}$ in the dark. Then, samples were washed with washing buffer (blocking buffer diluted 1:5 in PBS) and incubated overnight at $4{ }^{\circ} \mathrm{C}$ with the secondary antibody, biotin-conjugated, rabbit anti-mouse IgG1 (1:1000; Sigma Aldrich). After washing, samples were incubated for $1 \mathrm{~h}$ with streptavidin Alexa FluorTM 647 conjugate (1:200; Fisher Scientific), followed by washing thrice with washing buffer. All antibody dilutions were prepared with washing buffer (blocking buffer diluted 1:5 in PBS).

To visualize actin bundles, cells were stained with Alexa FluorTM 488 phalloidin (1:200 in PBS; Thermo Fisher Scientific) for $20 \mathrm{~min}$ at room temperature, followed by washing three times with PBS. To visualize cell nuclei, samples were incubated for 6 min with 4',6-Diamidin-2-phenylindol (DAPI, 1:70 in PBS; Sigma Aldrich) at room temperature, washed thrice with PBS and mounted with Mowiol ${ }^{\circledR}$ (Sigma Aldrich). Cells were imaged in with a Nikon Eclipse Ti-E microscope (Nikon Instruments Europe BV, the Netherlands) using an oil objective. Images were further processed and merged using ImageJ.

\subsubsection{MSN uptake, calcein loading and release}

Stability of the films in solution was assessed by release experiments. To assess cellular uptake of MSNs and their loaded cargo from the films, hMSCs were seeded on spin-coated cover slips with ATTO 633 core-labelled MSNs loaded with calcein. Uncoated, $\mathrm{O}_{2}$ plasma surface-activated cover slips were used as a negative control. hMSCs cultured on tissue-culture plastic with addition of calcein-loaded MSN to the medium $(35 \mu \mathrm{g} / \mathrm{ml})$ were used as a positive control. Seeding densities were varied as follows for the different time points in order to obtain similar numbers of cells at the time of harvest and measurement $-2 \mathrm{~h}$ and $6 \mathrm{~h}$ : 10,000 cells/ $\mathrm{cm}^{2} ; 1$ d: 7500 cells $/ \mathrm{cm}^{2} ; 2$ d: 5000 cells $/ \mathrm{cm}^{2} ; 7$ d: 3000 cells/ $\mathrm{cm}^{2}$. Cellular uptake was measured using flow cytometry (Accuri B6 flow cytometer, BD Biosciences, US). Negative controls were used to gate living cells (P1). Samples were analysed aiming at 10,000 events within P1.

To determine the release kinetics of calcein dyes from MSNs and MSN-DOPCs in suspension and from films, MSNs were loaded by suspending them in a $25 \mu \mathrm{M}$ aqueous calcein solution at a concentration of $0.5 \mathrm{mg} / \mathrm{ml}$ and stirring for $3 \mathrm{~h}$ in the dark. Thereafter, MSNs and MSN-DOPCs were collected and washed once with bidistilled water before spin-coating. Supernatant and washing solutions were collected to determine the amount of loaded cargo using a Cary 300 fluorometer (Agilent Technologies, US).

Calcein AM blue release from MSNs and MSN-DOPC in suspension was monitored using a fluorometer. Calcein-loaded MSNs and MSN-DOPCs ( $1 \mathrm{mg}$ ) were suspended in $200 \mu \mathrm{l}$ PBS in a membranecontaining insert, placed in a cuvette and dialysed against $3.2 \mathrm{ml}$ PBS. Fluorescent signal intensity was measured at $435 \mathrm{~nm}$ over $34 \mathrm{~h}$ at room temperature to assess release kinetics. Release of calcein from MSN and MSN-DOPC films was measured by incubating spin-coated MSN and MSN-DOPC films in $2 \mathrm{ml}$ PBS under dynamic conditions. At 1 and $2 \mathrm{~d}$ for MSN films and $2 \mathrm{~h}, 6 \mathrm{~h}$, and $1 \mathrm{~d}$ for MSN-DOPC films, aliquots of the supernatant were sampled and analysed for fluorescent signal intensity at $514 \mathrm{~nm}$.

\subsection{Dex loading}

To investigate the applicability of MSN films for drug delivery in a biological setting, the particles were loaded with the osteogenesis-stimulating drug dexamethasone (Dex) prior to spin-coating. Loading of Dex was performed by suspending $1 \mathrm{mg}$ MSNs in a $200 \mu 10 \mathrm{mM}$ Dex solution in ethanol. After stirring overnight, MSNs were collected by centrifugation and suspended in bi-distilled water for spin-coating. Uncoated, $\mathrm{O}_{2}$ plasmaactivated cover slips and unloaded MSN films served as controls. Samples were washed shortly with PBS to avoid premature drug release before transfer into sterile plates for cell experiments. hMSCs were seeded on top of the MSN films at a density of 4000 cells $/ \mathrm{cm}^{2}$. Cells on Dex-loaded MSN films were either cultured in basic medium and all media was refreshed, in basic medium but only half of the medium was refreshed each time (basic $50 \%$ ) or in medium enriched with $10 \mathrm{nM}$ Dex (osteogenic medium) and all media was refreshed. Cells on unloaded MSN films were cultured in basic medium to investigate the effect of the MSNs themselves on osteogenic differentiation and all media was refreshed. Control cover slips were either cultured in basic medium (negative control) or osteogenic medium (positive control) and all media was refreshed. Medium was refreshed every 3-4 days.

\subsection{ALP assay}

Osteogenic differentiation was evaluated by measuring alkaline phosphatase (ALP) levels at day 7, 14 and 21 of culture. CyQuant ${ }^{\circledR}$ Cell Proliferation Assay Kit (Thermo Fisher Scientific) was used to measure DNA content for normalization of ALP levels. To measure ALP levels and DNA content, cells were lysed with cell-lysis buffer (provided with the kit, 1:20 in PBS) containing 0.1\% (vol/vol) RNAse A (Thermo Fisher Scientific) and three cycles of freezing-thawing at $-80^{\circ} \mathrm{C}$. First, medium was discarded, and cell layers were washed once with PBS. After freezing-thawing for $30 \mathrm{~min}$, RNAse lysis buffer was added to each well, and samples were frozen-thawed for two cycles of 30 min each. Once thawed, more RNAse lysis buffer was added, and samples were sonicated for $5 \mathrm{~min}$. To assure complete lysis of the cells, samples were then incubated for $60 \mathrm{~min}$ at room temperature.

ALP activity was quantified using CDP-star solution (ready-touse, Sigma Aldrich). Cell lysate was incubated 1:5 with the reagent for $30 \mathrm{~min}$ in the dark at room temperature in a white-bottom, 96well plate. Luminescent signal was read on a spectrophotometer. ALP values were normalized with total DNA content per sample and expressed as an $\mathrm{x}$-fold increase compared to the negative control.

For the quantification of total DNA content, cell lysate was mixed with GR-dye solution (provided in the CyQuant kit, 1:200 in lysis buffer) according to the supplier's instructions. After $15 \mathrm{~min}$, fluorescent signal was measured with a spectrophotometer at $520 \mathrm{~nm}$. Absolute amount of DNA was calculated using the standard curve prepared following the supplier's instructions.

\subsubsection{Mineralization assay}

Osteogenic differentiation of hMSCs on MSN films was further assessed by Alizarin Red S (sodium alizarin sulphonate) staining to visualize mineralization. For this purpose, cells were seeded on Dex-loaded MSN films, unloaded MSN films or $\mathrm{O}_{2}$ plasmaactivated cover slips as described above. Cells on Dex-loaded 
MSN films were either cultured in basic medium with addition of $10 \mathrm{mM} \beta$-glyerolphosphate (BGP) referred to as BGP medium, in BGP medium but only half of the medium was refreshed each time (BGP 50\%) or in mineralization medium (basic medium with addition of $10 \mathrm{nM}$ Dex and $10 \mathrm{mM}$ BGP). Cells on unloaded MSN films were cultured in phosphate medium to investigate the effect of Dex loading. Control cover slips were either cultured in basic medium (negative control) or mineralization medium (positive control) where all medium was refreshed every 3-4 days. After $28 \mathrm{~d}$ of culture, cells were fixed with $4 \%$ PFA, and calcium deposits were made visible by Alizarin Red S staining.

In short, Alizarin Red S was dissolved in bi-distilled water at a concentration of $22 \mathrm{mg} / \mathrm{ml}$. Once the solid was completely dissolved, pH was adjusted to 4.2 with $25 \%$ ammonium hydroxide, and the solution was filtered through a $0.45-\mu \mathrm{m}$ membrane. Prior to staining, cells were rinsed once with PBS followed by washing twice with bi-distilled water. Alizarin Red solution was filtered once more using a $0.45-\mu \mathrm{m}$ membrane before addition to the samples. The completely immersed samples were incubated for $2 \mathrm{~min}$ at room temperature followed by washing thrice with bi-distilled water. Staining was assessed by visual inspection.

\subsection{Data analysis}

Flow cytometry data was analysed using FlowJo software, Version 10 (FlowJo LLC, US). Microscopy images were processed using NIS software for background correction and ImageJ for merging channels. Statistical analysis was performed using IBM $^{\circledR}$ SPSS $^{\circledR}$ software, Version 25, (IBM corporate, US). Significant differences between groups were examined using non-parametric KruskalWallis test with Mann-Whitney post-hoc test and Bonferroni correction after assessment of data distribution and homogeneity of variances with Shapiro-Wilk test and Lavene's test, respectively. For comparison of two groups only, Mann-Whitney $U$ test was used. Statistical significance was denoted as ${ }^{*} \mathrm{p}<0.05,{ }^{* *} \mathrm{p}<0.01$, and ${ }^{* * *} \mathrm{p}<0.001$. All data is reported as mean \pm standard error of the mean (SEM).

Cells stained with phalloidin and DAPI were used for quantitative analysis of the cell morphology, in terms of cell area (measured by the number of pixels occupied) and form factor (numbers closer to 1 describes rounder cells). Segmentation and single-cell morphological measurements were performed by combining Image for image processing, Cell Profiler for segmentation and cell tracking [27] and Matlab ${ }^{\circledR}$ 2016b (Mathworks) for data analysis (grouping, matching and plotting the quantitative data generated from each image dataset). A schematic of this data analysis process is shown in Scheme S1.

\section{Results}

\subsection{MSN-based films are homogeneous and stable}

Spherical mesoporous silica nanoparticles (MSNs) with thiolfunctionalized cores and amine-functionalized surfaces were synthesized based on a previously reported co-condensation method [25]. The thiol groups allowed covalent coupling of fluorescent dyes without interfering with surface chemistry. SEM imaging confirmed the synthesis of spherical and evenly shaped MSNs with a diameter of $80 \mathrm{~nm}$ (Fig. 1A) and TEM analysis showed their mesoporous structure (Fig. 1B). Presence of the surface (amine) and core (thiol) functionalities was confirmed by fluorescent labelling with ATTO633 maleimide and FITC-NHS coupled to the thiol and amine groups of MSNs, respectively (Fig. 1C). Amine groups on the surface of the MSNs resulted in a positive surface charge as confirmed by zeta potential measurements $(40.7 \pm 0.5 \mathrm{mV})$. The MSNs had a polydispersity index (PdI) of $0.13 \pm 0.01$, showing that the particles were monodisperse.

To create nanoparticle-based films, spin-coating of MSN suspensions in ethanol was performed on surface-activated glass cover slips. SEM imaging revealed the deposition of continuous layers of nanoparticles with a homogeneous surface structure (Fig. 1D). The film thickness distribution was even over the glass slide and measured $95 \pm 3 \mathrm{~nm}$, determined by 3D laser scanning microscopy (Fig. S1A). The films were stable in aqueous conditions; no significant MSN diffusion from the films could be detected after $7 \mathrm{~d}$ and $14 \mathrm{~d}$ of incubation in PBS (Table S1) and films remained intact (Fig. S1B).

Next, MSN film stability under cell culture conditions was investigated by determining MSN uptake in hMSCs, measured by fluorescently labeled thiols present in the MSN core (Fig. 1E). After $1 \mathrm{~d}, 5.4 \pm 0.5 \%$ of hMSCs had taken up MSNs from the films compared to $22.9 \pm 2.9 \%$ of hMSCs when MSNs were added as a suspension (Fig. 1E). No change was observed after $2 \mathrm{~d}$, with $6.5 \pm 0.3 \%$ MSN-positive cells when cultured on films compared to $23.4 \pm 2.0 \%$ when added in suspension. This remained stable after $7 \mathrm{~d}(3.94 \pm 0.89 \%)$ and did not increase significantly after $14 \mathrm{~d}$ ( $8.5 \pm 1.71 \%$, Table S2), indicating that MSN films were stable under cell culture conditions.

\subsection{MSN-based films are biocompatible and support cell adhesion}

Having established that the functionalized MSN films were homogeneous and stable under cell culture conditions, we investigated the biocompatibility and the ability of hMSCs to attach and proliferate on MSN films. hMSCs were chosen as they are intensively investigated in tissue regenerative strategies due to their multipotency (inherent ability to differentiate into multiple lineages) and ease of isolation. To test biocompatibility of the MSN films in the short term, cell viability was determined for hMSCs cultured on MSN films after 1, 5 and 7 days using the LDH assay. No difference in extracellular LDH levels was observed between cells cultured on the films compared to controls, suggesting no negative effect of the MSN films on cell viability (Fig. 2A). To validate that culturing hMSCs on MSN films did not affect cell numbers at later time-points, DNA was quantified after 7, 14 and 21 days. The DNA content was comparable on MSN films and control glass slides at 7d of culture, demonstrating similar proliferation rates of hMSCs cultured on films or glass (Fig. 2B). For both conditions, a significant increase in cell numbers was observed after $14 \mathrm{~d}$ of culture due to proliferating cells. A small but significant difference in DNA content was found between the samples at $14 \mathrm{~d}$. However, no significant difference was observed between the samples on day 7 or 21, indicating that it had a neglible effect on overall cell number. Furthermore, cell numbers did not significantly increase after $14 \mathrm{~d}$ for either hMSCs cultured on the films or on control slides, indicating that the hMSCs reached confluency after this time in both conditions slowing down proliferation rates.

Given the impact of hMSC cell attachment in guiding cellular differentiation through the formation of focal adhesions $[2,28,29]$, cell adhesion of hMSCs on MSN films was investigated using SEM and vinculin antibody staining. Cell attachment to the extracellular matrix or other substrates mainly occurs through the formation of stress fibres and clustering of different focal adhesion proteins (including vinculin), followed by the maturation of these contact points [2]. hMSCs cultured on MSN films for 2 days showed cell-typical morphology with extended filopodia where the films remained intact (Fig. 2C). The formation of focal adhesions was also observed using fluorescence microscopy and vinculin staining (Fig. 2D, E). No differences could be observed in the number or extent of bundling of actin to form stress fibres in hMSCs grown on MSN films or control glass after 2 (Fig. 2D) or 

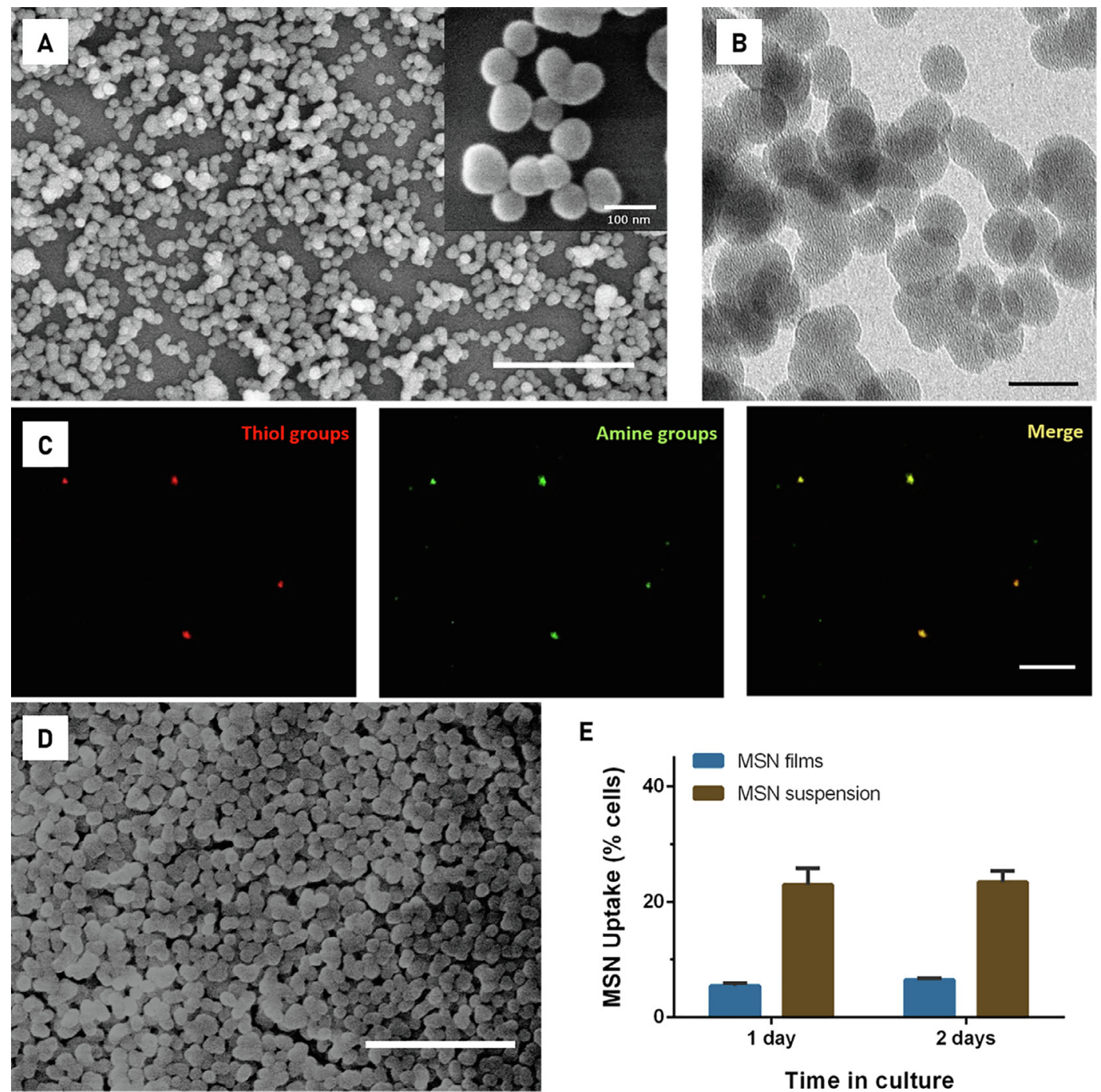

E

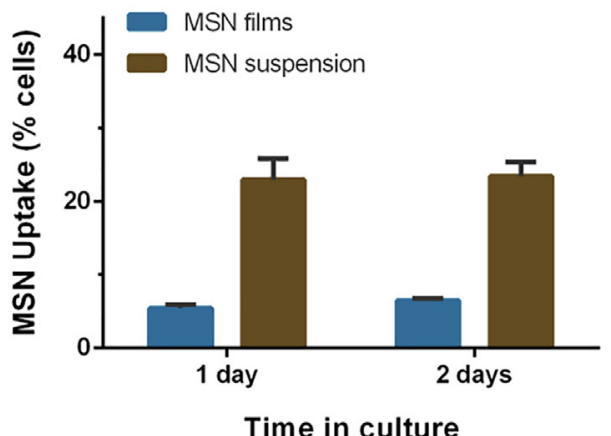

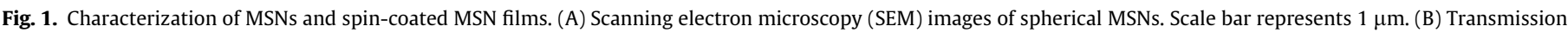

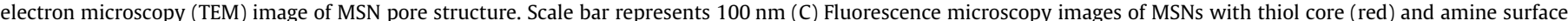

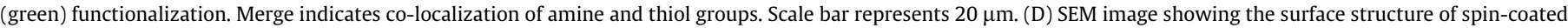

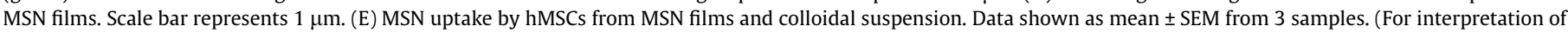
the references to colour in this figure legend, the reader is referred to the web version of this article.)

5 days (Fig. 2E). These results indicate that MSN-based films are biocompatible and support hMSC proliferation and cell adhesion similar to hMSCs grown on control surfaces.

\subsection{MSN films can deliver biomolecules to instruct stem cells}

MSNs have a high loading capacity and can efficiently encapsulate a variety of cargo, ranging from hydrophilic to hydrophobic drugs $[20,21,30]$. We thus tested the ability of the MSN films to store and deliver biomolecules, first using calcein-a cellpenetrable, fluorescent dye-as model cargo. To this end, calcein loaded MSNs were spin-coated on glass slides and calcein uptake by hMSCs was measured using flow cytometry. To compare calcein uptake between film and suspension conditions, calcein loaded MSNs were added to hMSCs cultures as a colloidal suspension (in equal amounts). Cellular uptake of calcein was 1.3-fold greater after $1 \mathrm{~d}$ (Figs. 3A and S2, left) and 2.6-fold greater after $2 \mathrm{~d}$ (Figs. 3A and S2, right) in hMSCs cultured on the MSN films compared to those exposed to the colloidal calcein loaded MSN suspension. The uptake of calcein by hMSCs when adding MSNs in suspension was stable over $2 \mathrm{~d}$, consistent with previous findings that MSNs in suspensions are internalized in hMSCs within the first few hours of culture [31].

Next, we tested the release of a relevant biomolecule from the MSN films-Dex, a synthetic glucocorticoid known to induce the expression of osteogenic marker genes in bone marrow cells $[32,33]$. Dex was selected as a model osteogenic factor, because its effects on alkaline phosphatase (ALP) production, an important osteogenic marker, are well studied [34]. hMSCs were cultured on glass slides under normal cell culture conditions (negative control, i.e. basic medium) and with $10 \mathrm{nM}$ Dex supplementation (positive control, i.e. osteogenic medium), and all media was exchanged every 3-4 days. For hMSCs cultured on MSN films the following conditions were included; MSN (hMSCs cultured on MSN-film, $100 \%$ basic media exchange every 3-4 days), MSN-Dex (hMSCs cultured on Dex-incorporated MSN-film, 100\% basic media exchange every 3-4 days), MSN-Dex 50\% (hMSCs cultured on Dexincorporated MSN-film, 50\% basic media exchange every 34 days), MSN-Dex osteo (hMSCs cultured on Dex-incorporated MSN-film, 100\% osteogenic media exchange every 3-4 days). ALP levels were measured to determine the efficacy of Dex released from the films on osteogenic differentiation of hMSCs films. 
A
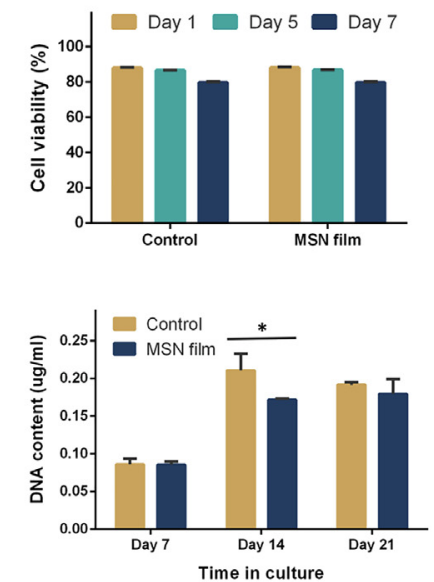

D
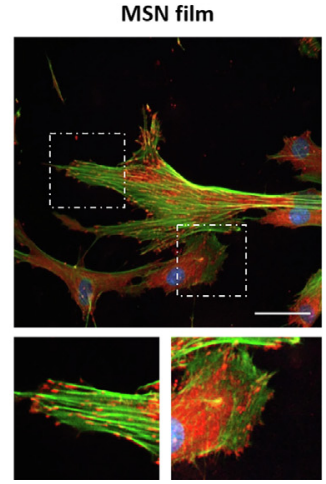

c
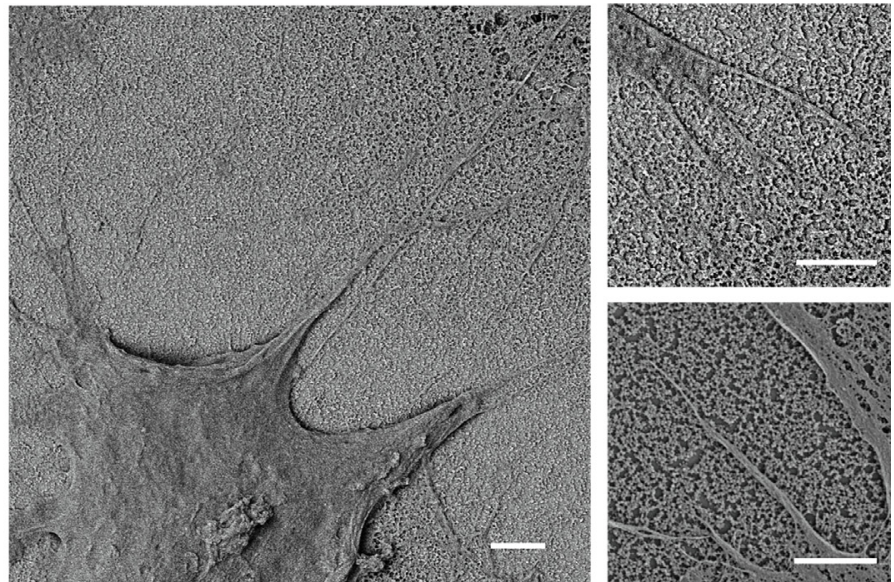

Flat control

E
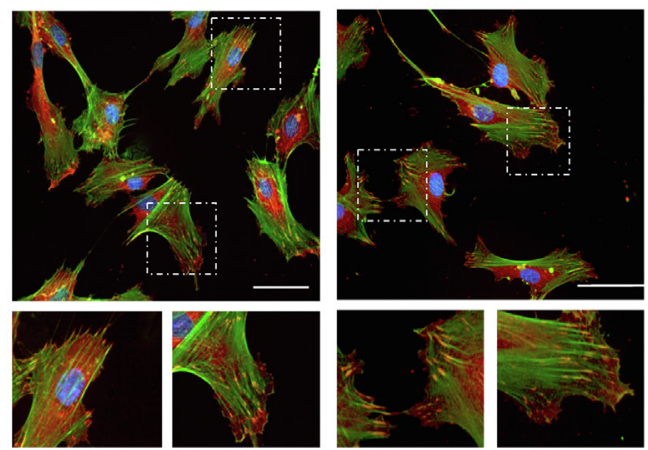
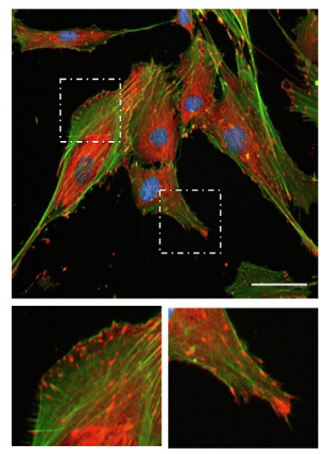

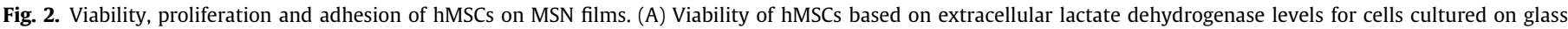

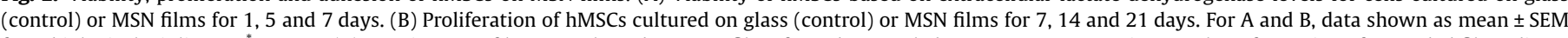

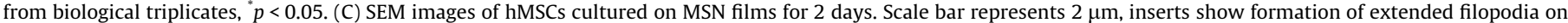

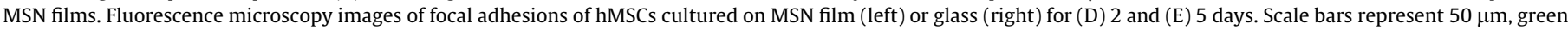

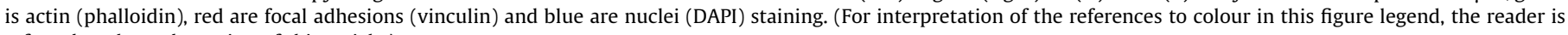
referred to the web version of this article.)

The ALP level was 4.4-fold higher $(\mathrm{p}<0.01)$ after $7 \mathrm{~d}$ and 7.1fold higher $(p<0.01)$ after $14 \mathrm{~d}$ for hMSCs cultured on Dexloaded MSN films (and 50\% refreshed medium) compared to those cultured on negative controls (Fig. 3B). These ALP levels were also higher compared to the positive control, Dex added to the medium (osteogenic medium), which showed 3.9-fold ( $p<0.01$ ) and 5.8fold ( $p<0.01$ ) after 7 and $14 \mathrm{~d}$ of culture, respectively. No significant ALP increase was found on Dex-loaded MSN films where all media was exchanged every 3-4 days (removing all released Dex), compared to the negative control.

In addition to ALP levels, we assessed matrix mineralization, a crucial step for the formation of hard tissues and considered the last stage of osteoblast differentiation [35]. Matrix mineralization was determined by staining calcium deposition in hMSCs after $28 \mathrm{~d}$ of culture. Mineralization could be observed in the positive controls which consisted of hMSCs cultured on glass substrates in the presence of BGP- and Dex- supplemented media which was fully replenished every 3-4 days (i.e. mineralization medium). MSN films that incorporated Dex where the media was fully exchanged every 3-4 days resulted in comparable mineralization as observed in the positive controls. However, similar to our previous observation concerning the ALP levels, the strongest mineralization was found on Dex-loaded MSN films when half of the medium was refreshed every 3-4 days, which was also substantially higher than positive controls (Figs. $3 \mathrm{C}$ and S3).

In summary, MSN films loaded with Dex resulted in higher ALP levels in hMSCs and increased mineralization compared to positive controls where Dex was added to the medium.

\subsection{MSN-DOPC films affect cell morphology}

To determine whether we could modulate cargo release from the MSN films, surface modification of MSNs with SLB was investigated. Next to good biocompatibility and low toxicity, SLB have been shown to improve cargo retention and facilitate stimuli-responsive release [26,36]. In addition to functioning as gate-keepers, SLBs are also a promising platform to create dynamic biological interfaces to control cell behaviour. In particular, where the head group of the lipid determines the interaction with cells, the alkyl chain composition defines the phase behaviour of the SLB (i.e. mobility of the lipids within the SLB) [24]. The formation of SLB around MSN is based on a solvent-exchange method: the water content of an ethanolic lipid monomer solution is gradually increased, resulting in the self-assembly of a lipid bilayer on the solid MSN surface [23,26]. Here we used 1,2-dioleoyl-sn-glycero3-phosphocholine (DOPC) lipid to create MSN-DOPC, which consists of a zwitter ionic head group providing a protein-resistant surface, and a low melting temperature resulting in a mobile lipid layer. Successful SLB formation was confirmed by a decrease in zeta surface potential for DOPC-coated MSNs $(8.7 \pm 0.2 \mathrm{mV})$ compared to uncoated MSN $(40.7 \pm 0.5 \mathrm{mV})$, with a PdI of $0.30 \pm 0.01$.

The development of films using MSN-DOPC was only possible when spin-coating MSN-DOPC mixed with plain MSNs (ratio 1:1). Spin-coating MSN-DOPC particles alone would form aggregates on the glass and it was not possible to form homogeneous films. The continuous, MSN: MSN-DOPC (1:1) films showed a homogenous surface structure (Fig. 4A), with a thickness of 
A

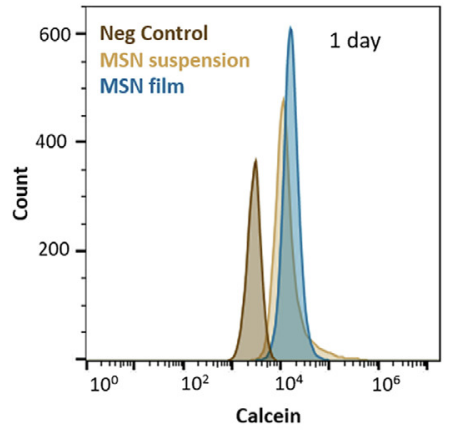

C

Neg Control

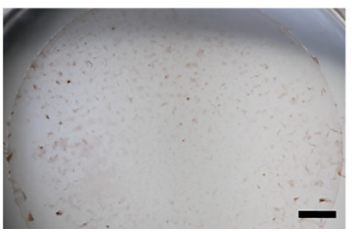

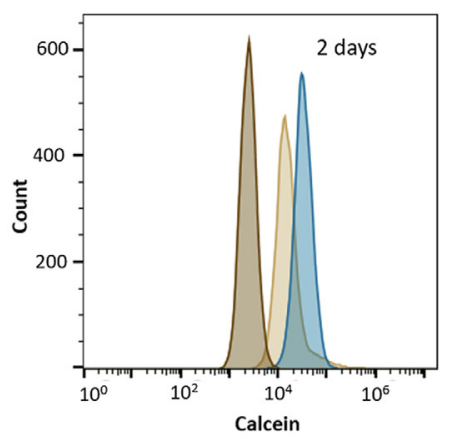

BGP

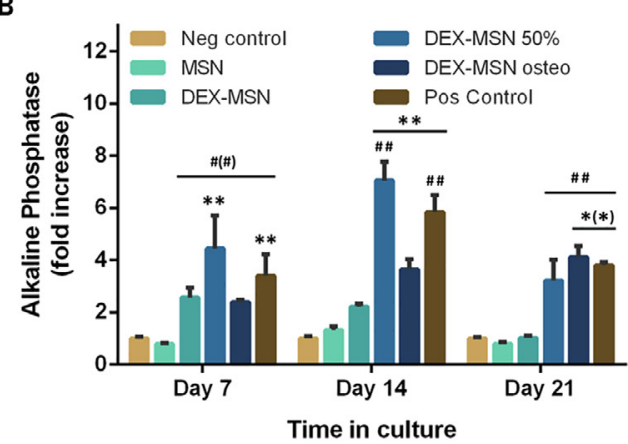

BGP $50 \%$
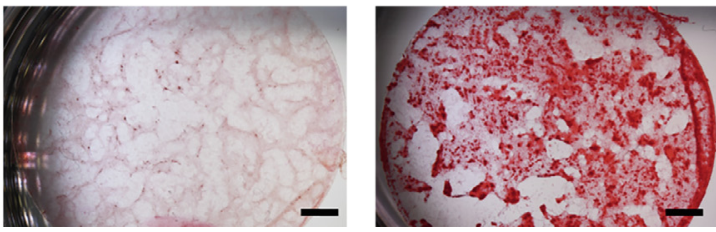

Pos Control

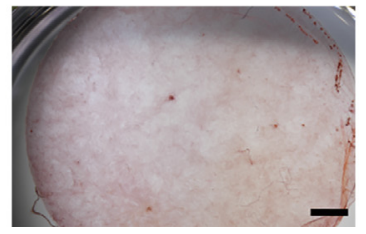

Fig. 3. Drug delivery capacity of MSN films to induce osteogenic differentiation in hMSCs. (A) Histograms of calcein uptake, determined by fluorescence intensity, from hMSCs cultured on MSN films, or with MSN suspensions. As a negative control, hMSCs were cultured on glass without MSNs. (B) Alkaline phosphatase production of hMSCs cultured on dexamethasone-free (MSN) or dexamethasone-loaded MSN films (DEX-MSN) in basic medium. DEX-MSN 50\% refers to refreshing half of the medium. Controls were cultured on glass in basic (negative control) or dexamethasone-supplemented osteogenic medium (positive control), with full media replenishment every 3-4 days. Values are $x$-fold increases compared to the negative control. Data is shown as mean \pm SEM of biological triplicates. * Significant difference compared to neg control, \# compared to MSN basic. "p $<0.05 ; " p>0.01$. (C) Visualization of calcium deposits (red) by hMSCs after 28 days of culture. In experimental conditions, cells were cultured on dexamethasone-loaded MSN films with addition $\beta$-glycerolphosphate (BGP) to the medium where either all (BGP) or only half of the medium (BGP $50 \%$ ) was changed every 3-4 days. hMSCs were cultured on glass cover slips in basic medium (negative control) or mineralization medium containing dexamethasone and $\beta$ - glycerolphosphate (positive control), with full media replenishment every 3-4 days. Representative images of calcium deposits per condition are shown, scale bar represents $2 \mathrm{~mm}$. (For interpretation of the references to colour in this figure legend, the reader is referred to the web version of this article.)

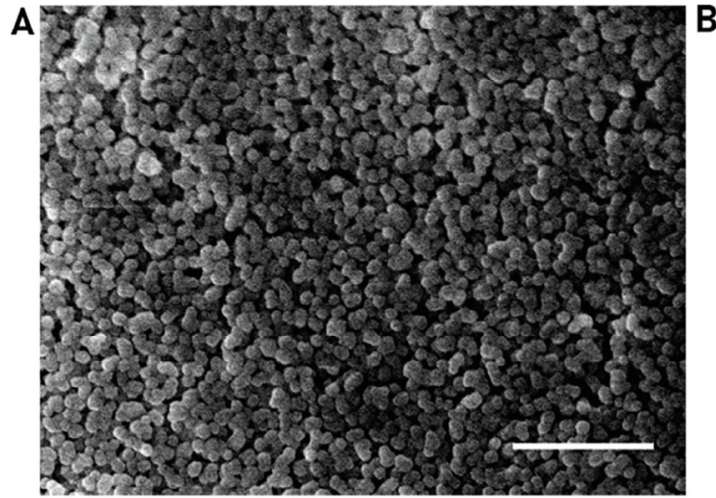

C

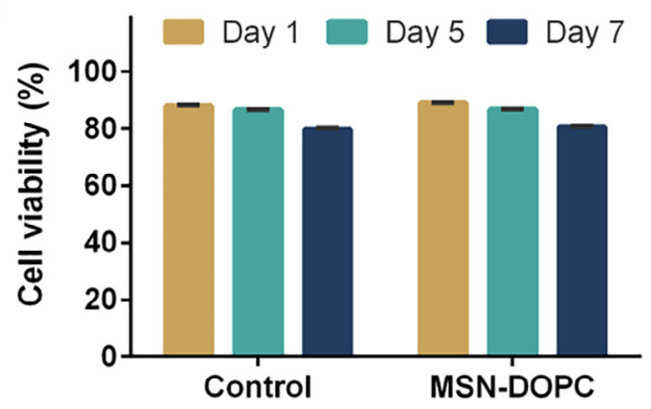

B

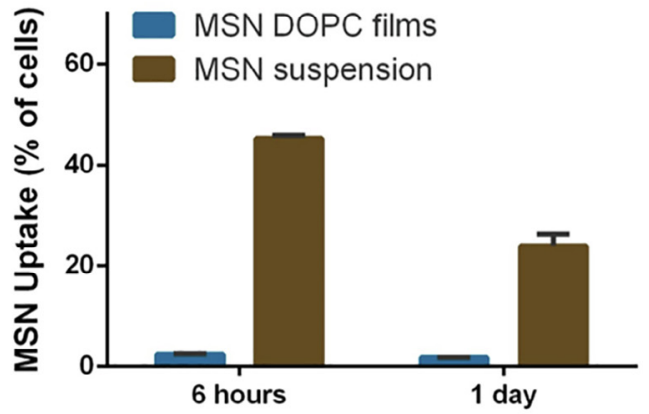

Time in culture

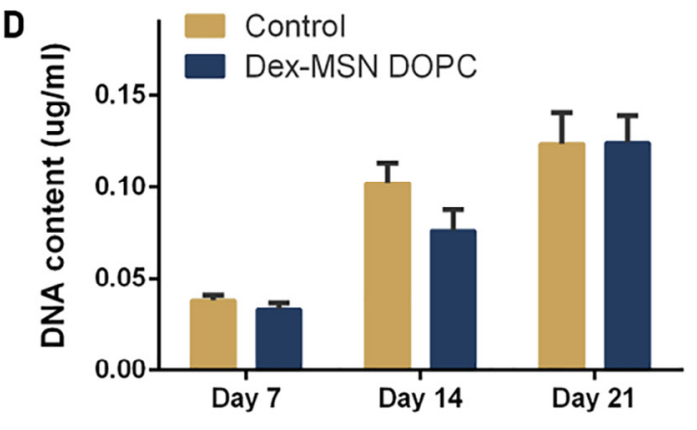

Time in culture

Fig. 4. MSNs films with supported lipid bilayer (SLB) surface functionalization. (A) SEM image showing the surface structure of SLB-modified (DOPC) MSN films. Scale bar represents $1 \mu \mathrm{m}$. (B) MSN uptake by hMSCs from MSN-DOPC films and in a colloidal suspension. (C) Viability, based on extracellular lactate dehydrogenase levels, of hMSCs cultured on glass (control) or MSN-DOPC films for 1, 5 and 7 days. (D) Proliferation of hMSCs cultured on glass (control) or MSN-DOPC films for 7, 14 and 21 days. For panels $\mathrm{B}-\mathrm{D}$, data shown as mean \pm SEM biological triplicates. 



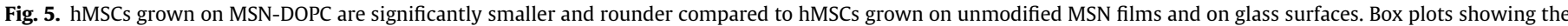

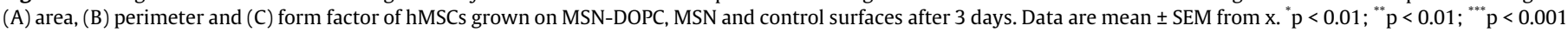
Upper border and lower border of the boxes are third quartile and first quartile accordingly. + are mean of each data group; ns is non-significant.

$105 \pm 4 \mathrm{~nm}$ (Fig. S4A and B). When fluorescently labelling the MSNDOPC before spin-coating, small pockets of MSN-DOPC nanoparticles could be detected, which were homogeneously dispersed (Fig. S4C). MSN-DOPC films were stable in PBS (Table S1, Fig. S4D) and hMSCs cultured on MSN-DOPC films showed very low uptake of MSNs also after 7 and 14 days of culture, demonstrating that the lipid surface modification did not affect film stability (Fig. 4B, Table S2). No difference in cell viability, measured by extracellular LDH levels, was observed between cells cultured on MSN-DOPC films and glass slide controls, indicating that the films were biocompatible (Fig. 4C). In addition, hMSCs cultured on MSN-DOPC films were viable and proliferated to a similar extent compared to hMSCs cultured on glass (Fig. 4D).

Next, we evaluated the effect of MSN-DOPC on hMSC cell shape factors. Cell area, cell perimeter and circularity (form factor approaches 1 for highly circular cells) were quantified and compared to cells grown on MSN films and control glass (Fig. 5). hMSCs grown on MSN-DOPC films showed a significant reduction in cell perimeter $(-21 \%)$ and cell area $(-15 \%)$ compared to hMSCs grown on control glass. A significantly higher form factor was found for hMSCs cultured on MSN-DOPC ( 0.24 vs. 0.18 for control surfaces, Fig. 5), indicating more circular cells. Cell perimeter and area were also reduced for hMSCs grown on MSN films, but the effect was much smaller: $-5 \%$ cell perimeter, $-11 \%$ cell area. The form factor of hMSCs grown on MSN films was similar to hMSCs cultured on control surfaces (0.18, Fig. 5C), demonstrating that the hMSCs maintained the same shape at a slightly smaller size. Thus, the addition of the SLB on MSN films resulted in smaller and rounder hMSCs.

\subsection{MSN-DOPC films release their cargo slower compared to MSN films}

To determine whether MSN-DOPC films, like MSN films, could release biomolecules, we incorporated calcein again as a model cargo in the MSN-DOPCs before spin-coating. As shown in Fig. $6 \mathrm{~A},<10 \%$ of calcein was released over $34 \mathrm{~h}$, with a dampened initial burst, from MSN-DOPC film compared to calcein release from MSN films. Additionally, hMSCs cultured on MSN-DOPC films took up less calcein compared to those cultured on MSN films, as seen in the median fluorescence intensity representing intracellular calcein concentration (Figs. 6B and S5). We found a 3.0-fold higher intensity from unmodified MSN films compared to DOPCmodified films at $2 \mathrm{~h}$, which continued for later time points, with a 3.6-fold and 2.0-fold higher intensity after $6 \mathrm{~h}$ and $1 \mathrm{~d}$, respectively. The same trend was seen when assessing the percentages of calcein-positive hMSCs. After $2 \mathrm{~h}$ of culture, only $12.8 \pm 3.9 \%$ of hMSCs cultured on SLB-modified MSN films had taken up calcein compared to $48.8 \pm 6.7 \%$ of hMSCs on MSN films. The percentage of calcein-positive cells increased to $25.1 \pm 5.1 \%$ and $65.8 \pm 15.8 \%$ on MSN-DOPC and MSN films, respectively, after $6 \mathrm{~h}$. After $1 \mathrm{~d}$, $77.9 \pm 8.5 \%$ of hMSCs on DOPC-modified MSC films had taken up calcein compared to $97.0 \pm 2.6 \%$ on unmodified MSN films. Next, Dex was incorporated in MSN-DOPC and spin-coated with nonloaded MSNs in a 1:1 ratio. The ALP production of hMSCs cultured on Dex-loaded MSN-DOPC films when all basic media was replenished every 3-4 days was compared to Dex-loaded on MSN films where again only $50 \%$ of the basic media was replenished every 3-4 days. Whereas after $7 \mathrm{~d}$ no significant difference could be detected (Fig. 6C), after $14 \mathrm{~d}$ a significant increase in ALP levels for hMSCs cultured on MSN-DOPC films (2.1-fold, p < 0.01) compared to negative controls (full media replenishment) was observed. However, these levels were lower compared to the positive control (4.6-fold, $\mathrm{p}<0.01$, full media replenishment) and to hMSCs cultured on Dex-incorporated MSN films where $50 \%$ of the media was replenished (6.4-fold, $\mathrm{p}<0.01$ Fig. S6C).

In summary, by using the SLB surface modification, calcein and Dex diffused more slowly from the MSN films, which resulted in significant ALP production.

\section{Discussion}

Here we report a nanostructured surface coating that offers high control over surface chemistry and which can simultaneously be used to deliver bioactive factors. It is well documented that surface structure (topography) can be used to control important cellular and molecular events, for example, to promote adhesion and integration of the surrounding tissue $[1-3,37,38]$. Moreover, the use of soluble factors has been a popular approach to improve the bioactivity of synthetic biomaterials [9-11]. Our approach leverages the drug delivery capabilities of MSNs; their porous structure can be used to incorporate different types of cargo, while their surface can be easily modified to tune their release kinetics $[12,13]$. These features are combined with their promising surface characteristics, which support cell adhesion and proliferation but can also be used to chemically modify the surface to provide surface cues (e.g. to modulate cell shape). Although here we used Dex as a soluble factor and lipid bilayers as surface modifications, many other types of cargo and surface modifications can be used.

To create the films, core- and surface-functionalized $(80 \mathrm{~nm}$ sized) MSNs were synthesized and spin-coated on (plasmatreated) glass slides to create stable homogeneous films. The films were also stable under cell culture conditions, i.e. hMSCs cultured on the films did not significantly internalize MSNs. Moreover, DOPC lipid surface modifications did not affect film stability. These findings are in contrast with a study by Bocking et al. [39], who reported prominent cell uptake of MSNs from films after $24 \mathrm{~h}$. This 
A

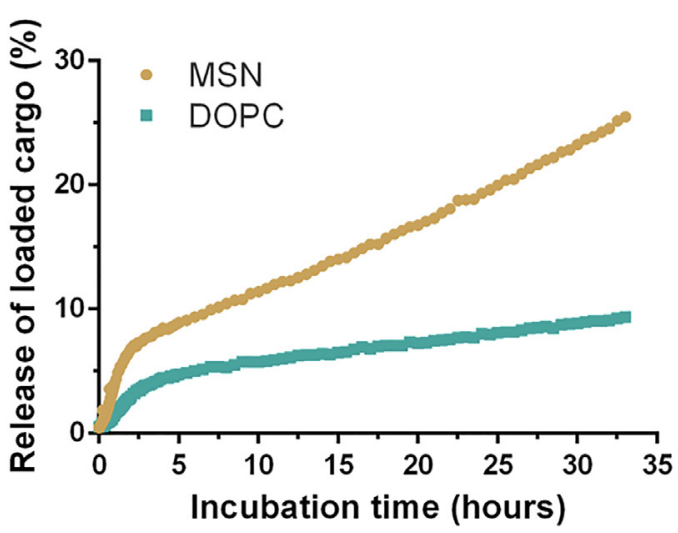

C

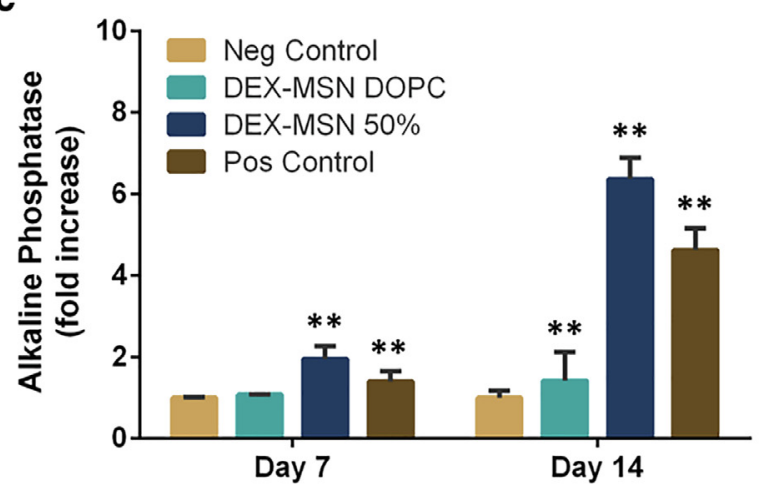

Time in culture
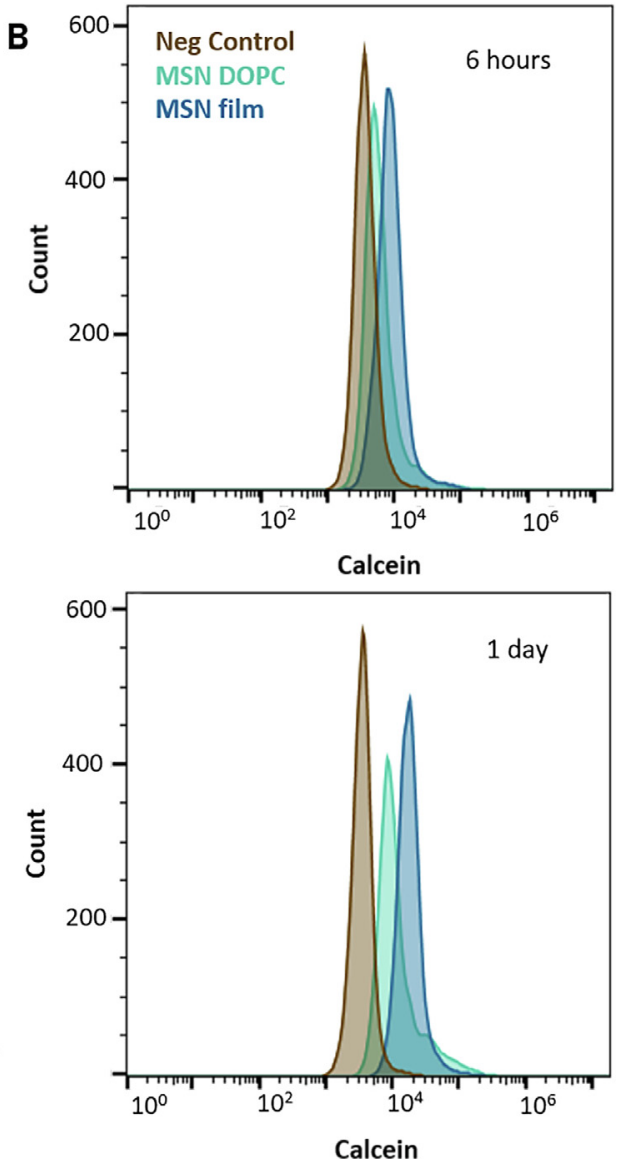

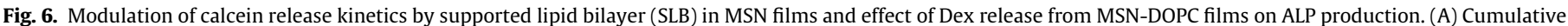

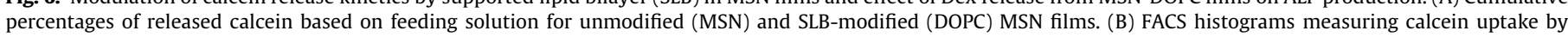

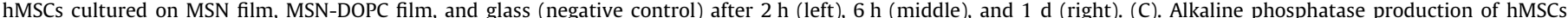

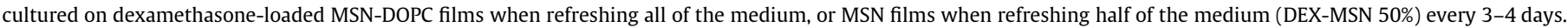

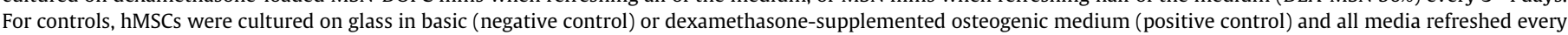

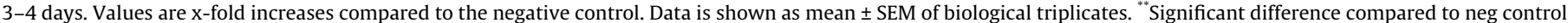
$(\mathrm{p}<0.01)$.

may be due to differences in nanoparticle properties and preparation of MSN films between the two studies. First, our MSNs are surface-functionalized with positively charged amines, whereas the Bocking study utilized non-surface-modified MSNs. The positively charged amines would give the MSNs a strong affinity to the negatively charged, $\mathrm{O}_{2}$ plasma-treated spinning substrate, thus yielding more stable MSN films. Second, a different cell type (C2C12 myoblasts) was used, which may have more permissive uptake routes compared to the hMSCs used here. The benefit of a stable film as developed here is the locally retained drug delivery (near the film), and sustained drug release (slow diffusion from MSN pores) without effecting coating integrity. However, their surface chemistry can easily be adjusted to create less stable films (e.g. by lowering MSN surface zeta potential) to also allow nanoparticle delivery in cells, as previously demonstrated by Bocking et al. [39].

hMSCs cultured on MSN films showed cell-typical morphology with extended filopodia, and formed focal adhesion points to a similar extend as hMSCs grown on glass surfaces. Also, no significant influence on cell shape (form factor) could be observed for cells grown on MSN films and flat controls. This is in line with literature; contact guidance (the instruction of cells to align or spread in a certain shape by surface topography) has mainly been reported for micro-sized grooves or pillars [1-3]. Thus, MSN films can serve as a biocompatible cell-adherent nanomaterial without affecting cell shape, cell adhesion or proliferation rates. The cell-adhesive properties of MSNs have already been documented in other systems: MSN addition to hydrogel networks has led to improved stem cell adhesion, migration and spreading [17-19].

MSN surface functionalization with lipids (DOPC), did significantly affect hMSC cell shape and size, hMSCs cultured on MSN: MSN-DOPC (1:1) films were significantly smaller and rounder compared to hMSCs cultured on glass slides or pure MSN-films. The antifouling properties of PC lipids like DOPC are well known and are attributed to the zwitterionic lipid head group [24]. Previously, it has been shown that SLBs made from DOPC on a flat surface (so not on nanoparticle surfaces) prevented stem cell adhesion [38]. Here, the 1:1 mix of cell-adherent surface (MSN) and cell-resistant surface (MSN-DOPC) did not interfere with hMSC cell number or proliferation rate but did allow for the modulation of stem cell shape and size. This is likely due to less available cell adhesion sites on the MSN: MSN-DOPC surface compared to the MSN-films. SLB nanoparticle surface modification thus seem to be an interesting approach to control hMSC adhesion and spreading on nanoparticle-based films. Moreover, cell shape has been shown to be an important regulator of stem cell differentiation, where hMSCs cell spreading has been linked with osteogenesis [37,38]. Future studies should investigate whether MSN-supported lipid films as the ones proposed here can have a similar effect in directing stem cell fate by modulating cell shape.

The porous structure of the MSNs allowed efficient encapsulation of calcein dye and Dex, a synthetic glucocorticoid known to 
induce the expression of osteogenic marker genes. Calcein delivery from the films was more efficient compared to administration of the same amount of MSNs as a suspension. Since we observed co-localization of calcein and MSN particles, it is likely that calcein uptake is directly related to MSN uptake when MSNs are added as a suspension. In contrast, calcein can diffuse from the MSN films, leading to locally higher calcein concentrations. Thus, hMSCs in direct contact with the MSNs in the MSN films are exposed to higher cargo concentrations. The higher local cargo concentrations near the film and thus near the adhered cells, could also explain the several fold increase in ALP signal and a significantly higher mineralization when Dex was incorporated in the film, compared to positive control conditions (i.e. Dex directly added to the medium). ALP production and mineralization has been shown to be Dex concentration-dependent [40], indicating that the higher local Dex concentrations when using Dex-loaded MSN-films may lead to improved bone forming potential of hMSCs. Efficient drug delivery from stable MSN-based coatings has previously been reported for the delivery of small molecule RAPA, which led to a higher reendothialization in vivo compared to RAPA incorporating polymer controls [41].

However, increased ALP levels and mineralization was only observed when $50 \%$ of the medium was exchanged compared to control conditions where all medium was exchanged. Replenishing media every 3-4 days is needed because of the long culture conditions (up to 21 days for ALP and up to 28 days for mineralization assays). Whereas in the positive controls Dex is presented to the cells as a homogeneous solution, in the case of the MSN-films, Dex is locally released from the films. Thus, when fully exchanging media, released Dex from the MSN-film was completely removed every 3-4 days, indicating that Dex release was not sustained over the full 21 days period. Since the in vitro differentiation of hMSCs towards an adult lineage relies on continuous presence of bioactive molecules, we pursued the possibility of using lipid bilayer surface functionalization to slow down cargo release. The lipid surface modification has been used by us and others to create biocompatible MSNs that allow sustained cargo release by blocking the MSN pores [23,26,31]. Indeed, we could show that using a lipid bilayer, calcein delivery was significantly slowed down; both from the films and when hMSCs were cultured on MSN-DOPC films. Especially the initial burst release could be dampened when using DOPC, and release of calcein over several days was significantly slowed down. The slower release of Dex from MSN-DOPC also led to a significant increase in ALP levels after 14 days when all media was removed every 3-4 days, albeit lower compared to positive controls and Dex-incorporated MSN films where only $50 \%$ of the media was replenished. Future studies should be directed into investigating the potential of such coatings in vivo where the local Dex concentrations are likely to remain higher. Thus, surface modifications like SLB can help to provide a more sustained cargo release which may better mimic the spatiotemporal appearance of biological cues during regeneration and improve the bioactivity of synthetic materials.

\section{Conclusions}

In summary, MSNs can be used to create an adherent biocompatible and stable nanostructured surface that can be chemically modified to instruct cell behaviour both by providing surface cues and soluble cues. As far as we are aware, our study is the first one to prepare stable MSN-based films for sustained release of cargo, where the release could be modulated by using lipid surface modifications.

Specifically, we demonstrated efficient local Dex delivery from mesoporous silica nanoparticle-based films to direct hMSC differentiation towards the bone lineage without affecting coating integrity. The stable MSN films supported hMSC cell adhesion and proliferation, and Dex delivery from the MSN films resulted in increased levels of osteogenic markers compared to MSN- or Dex-supplemented medium. In addition, we demonstrate that SLB surface modification of the nanoparticles allowed the possibility to modulate release kinetics from the films, offering better temporal control over Dex release. SLB surface modifications were possible without affecting film integrity, cell adhesion or proliferation rates. However SLB surface modifications did affect cell morphology (size, shape and form factor), representing an interesting approach to control hMSC adhesion and spreading on nanoparticle-based films using surface cues. In addition, nanoparticle surface modifications such as SLB offer interesting ways for sequestered delivery of multiple cargo's to generate coatings that can enhance biomaterial performance by (tightly) guiding tissue formation. In particular, as both MSN types (SLB-modified and unmodified) can be mixed into the same MSN film, delivery of multiple cargos but also sequestrated release of the bioactive molecules may be possible.

In conclusion, MSNs are multi-functional tools for the design of material coatings with incorporated, sustained drug delivery to modulate cell behaviour. We envisage the application of these materials as implant coatings to improve tissue integration and thereby reducing healing time. For example, future applications for the developed MSN film include the coating of metal implants for joint replacement applications, a major orthopaedic procedure used to treat joint osteoarthritis. Implant failure is a common clinical problem caused by aseptic loosening and infection. The MSN films can impart bioactivity and biocompatibility to the metallic surface to promote bone ingrowth and differentiation of stem cells into osteoblasts leading to enhanced osteointegration.

\section{Acknowledgements}

We thank Dr. Carmen Lopez for technical assistance with TEM imaging and Denis Beurden for technical assistance in the cell biology assays. This research has been in part made possible with the support of the Dutch Province of Limburg (LINK), and the Gravitation research programme Materials Driven Regeneration with project number 024.003.013, which is partly financed by the Netherlands Organisation for Scientific Research (NWO).

\section{Appendix A. Supplementary material}

Supplementary data to this article can be found online at https://doi.org/10.1016/j.actbio.2019.07.008.

\section{References}

[1] M.J. Dalby, M.O. Riehle, S.J. Yarwood, C.D. Wilkinson, A.S. Curtis, Nucleus alignment and cell signaling in fibroblasts: response to a micro-grooved topography, Exp. Cell Res. 284 (2) (2003) 274-282.

[2] J.W. Cassidy, J.N. Roberts, C.A. Smith, M. Robertson, K. White, M.J. Biggs, R.O. Oreffo, M.J. Dalby, Osteogenic lineage restriction by osteoprogenitors cultured on nanometric grooved surfaces: the role of focal adhesion maturation, Acta Biomater. 10 (2) (2014) 651-660.

[3] Y. Yang, K. Wang, X. Gu, K.W. Leong, Biophysical regulation of cell behaviorcross talk between substrate stiffness and nanotopography, Engineering (Beijing) 3 (1) (2017) 36-54.

[4] M.J. Dalby, N. Gadegaard, R. Tare, A. Andar, M.O. Riehle, P. Herzyk, C.D. Wilkinson, R.O. Oreffo, The control of human mesenchymal cell differentiation using nanoscale symmetry and disorder, Nat. Mater. 6 (12) (2007) 997-1003.

[5] P. Boyne, S.D. Jones, Demonstration of the osseoinductive effect of bone morphogenetic protein within endosseous dental implants, Implant. Dent. 13 (2) (2004) 180-184.

[6] Y. Liu, K. de Groot, E.B. Hunziker, BMP-2 liberated from biomimetic implant coatings induces and sustains direct ossification in an ectopic rat model, Bone 36 (5) (2005) 745-757. 
[7] W. Cui, Q.Q. Liu, L. Yang, K. Wane, T.F. Sun, Y.H. Ji, L.P. Liu, W. Yu, Y.Z. Qu, J.W. Wang, Z.G. Zhao, J.T. Zhu, X.D. Guo, Sustained delivery of BMP-2-related peptide from the true bone ceramics/hollow mesoporous silica nanoparticles scaffold for bone tissue regeneration, Acs Biomater. Sci. Eng. 4 (1) (2018) 211 221.

[8] N. Ehlert, A. Hoffmann, T. Luessenhop, G. Gross, P.P. Mueller, M. Stieve, T. Lenarz, P. Behrens, Amino-modified silica surfaces efficiently immobilize bone morphogenetic protein 2 (BMP2) for medical purposes, Acta Biomater. 7 (4) (2011) 1772-1779.

[9] H.Y. Zhao, J. Wu, J.J. Zhu, Z.C. Xiao, C.C. He, H.X. Shi, X.K. Li, S.L. Yang, J. Xiao Research advances in tissue engineering materials for sustained release of growth factors, Biomed. Res. Int. 2015 (2015) 808202.

[10] L. Liu, X. Zhou, Y. Xu, W. Zhang, C.H. Liu, X. Wang, Controlled release of growth factors for regenerative medicine, Curr. Pharm. Des. 21 (12) (2015) 1627 1632.

[11] T.N. Vo, F.K. Kasper, A.G. Mikos, Strategies for controlled delivery of growth factors and cells for bone regeneration, Adv. Drug Deliv. Rev. 64 (12) (2012) 1292-1309.

[12] J.M. Rosenholm, V. Mamaeva, C. Sahlgren, M. Linden, Nanoparticles in targeted cancer therapy: mesoporous silica nanoparticles entering preclinical development stage, Nanomedicine (Lond.) 7 (1) (2012) 111-120.

[13] R. Narayan, U.Y. Nayak, A.M. Raichur, S. Garg, Mesoporous silica nanoparticles: a comprehensive review on synthesis and recent advances, Pharmaceutics 10 (3) (2018).

[14] M. Kato, N. Shoda, T. Yamamoto, R. Shiratori, T. Toyo'oka, Development of a silica-based double-network hydrogel for high-throughput screening of encapsulated enzymes, Analyst 134 (3) (2009) 577-581.

[15] R. Lach, G.M. Kim, G.H. Michler, W. Grellmann, K. Albrecht, Indentation fracture mechanics for toughness assessment of PMMA/SiO2 nanocomposites, Macromol. Mater. Eng. 291 (3) (2006) 263-271.

[16] S.B. Yang, J. Wang, H.L. Tan, F.Y. Zeng, C.S. Liu, Mechanically robust PEGDAMSNs-OH nanocomposite hydrogel with hierarchical meso-macroporous structure for tissue engineering, Soft Matter 8 (34) (2012) 8981-8989.

[17] B. Baumann, R. Wittig, M. Linden, Mesoporous silica nanoparticles in injectable hydrogels: factors influencing cellular uptake and viability, Nanoscale 9 (34) (2017) 12379-12390.

[18] F. Fiorini, E.A. Prasetyanto, F. Taraballi, L. Pandolfi, F. Monroy, I. LopezMontero, E. Tasciotti, L. De Cola, Nanocomposite hydrogels as platform for cells growth, proliferation, and chemotaxis, Small 12 (35) (2016) 4881-4893.

[19] H. Shokry, U. Vanamo, O. Wiltschka, J. Niinimaki, M. Lerche, K. Levon, M. Linden, C. Sahlgren, Mesoporous silica particle-PLA-PANI hybrid scaffolds for cell-directed intracellular drug delivery and tissue vascularization, Nanoscale 7 (34) (2015) 14434-14443.

[20] S. van Rijt, P. Habibovic, Enhancing regenerative approaches with nanoparticles, J. R. Soc. Interf. 14 (129) (2017).

[21] J.M. Rosenholm, J. Zhang, M. Linden, C. Sahlgren, Mesoporous silica nanoparticles in tissue engineering-a perspective, Nanomedicine (Lond.) 11 (4) (2016) 391-402.

[22] J. Wen, K. Yang, F. Liu, H. Li, Y. Xu, S. Sun, Diverse gatekeepers for mesoporous silica nanoparticle based drug delivery systems, Chem. Soc. Rev. 46 (19) (2017) 6024-6045.

[23] K.S. Butler, P.N. Durfee, C. Theron, C.E. Ashley, E.C. Carnes, C.J. Brinker, Protocells: modular mesoporous silica nanoparticle-supported lipid bilayers for drug delivery, Small 12 (16) (2016) 2173-2185.

[24] J. van Weerd, M. Karperien, P. Jonkheijm, Supported lipid bilayers for the generation of dynamic cell-material interfaces, Adv. Healthc. Mater. 4 (18) (2015) 2743-2779.
[25] V. Cauda, A. Schlossbauer, J. Kecht, A. Zurner, T. Bein, Multiple core-shell functionalized colloidal mesoporous silica nanoparticles, J. Am. Chem. Soc. 131 (32) (2009) 11361-11370.

[26] V. Cauda, H. Engelke, A. Sauer, D. Arcizet, C. Brauchle, J. Radler, T. Bein, Colchicine-loaded lipid bilayer-coated $50 \mathrm{~nm}$ mesoporous nanoparticles efficiently induce microtubule depolymerization upon cell uptake, Nano Lett. 10 (7) (2010) 2484-2492.

[27] A.E. Carpenter, T.R. Jones, M.R. Lamprecht, C. Clarke, I.H. Kang, O. Friman, D.A. Guertin, J.H. Chang, R.A. Lindquist, J. Moffat, P. Golland, D.M. Sabatini, Cell Profiler: image analysis software for identifying and quantifying cell phenotypes, Genome. Biol. 7 (10) (2006) R100.

[28] M.F. Griffin, M. Szarko, A. Seifailan, P.E. Butler, Nanoscale surface modifications of medical implants for cartilage tissue repair and regeneration, Open Orthop. J. 10 (2016) 824-835.

[29] B. Geiger, J.P. Spatz, A.D. Bershadsky, Environmental sensing through focal adhesions, Nat. Rev. Mol. Cell Biol. 10 (2009) 21.

[30] G.Y. Chen, I. Roy, C.H. Yang, P.N. Prasad, Nanochemistry and nanomedicine for nanoparticle-based diagnostics and therapy, Chem. Rev. 116 (5) (2016) 2826 2885.

[31] R. Rosenbrand, D. Barata, P. Sutthavas, R. Mohren, B. Cillero-Pastor, P. Habibovic, S. van Rijt, Lipid surface modifications increase mesoporous silica nanoparticle labeling properties in mesenchymal stem cells, Int. J. Nanomed. 13 (2018) 7711-7725.

[32] X. Zhou, W. Feng, K. Qiu, L. Chen, W. Wang, W. Nie, X. Mo, C. He, BMP-2 derived peptide and dexamethasone incorporated mesoporous silica nanoparticles for enhanced osteogenic differentiation of bone mesenchymal stem cells, ACS Appl. Mater. Interf. 7 (29) (2015) 15777-15789.

[33] Y. Chen, J.C. Li, N. Kawazoe, G.P. Chen, Preparation of dexamethasone-loaded calcium phosphate nanoparticles for the osteogenic differentiation of human mesenchymal stem cells, J. Mater. Chem. B 5 (33) (2017) 6801-6810.

[34] E.E. Golub, G. Harrison, A.G. Taylor, S. Camper, I.M. Shapiro, The role of alkaline phosphatase in cartilage mineralization, Bone Miner. 17 (2) (1992) 273-278.

[35] R.M. Porter, W.R. Huckle, A.S. Goldstein, Effect of dexamethasone withdrawal on osteoblastic differentiation of bone marrow stromal cells, J. Cell Biochem. 90 (1) (2003) 13-22.

[36] Q. Zhang, X. Chen, H. Shi, G. Dong, M. Zhou, T. Wang, H. Xin, Thermoresponsive mesoporous silica/lipid bilayer hybrid nanoparticles for doxorubicin on-demand delivery and reduced premature release, Colloids Surf. B Biointerf. 160 (2017) 527-534.

[37] R. McBeath, D.M. Pirone, C.M. Nelson, K. Bhadriraju, C.S. Chen, Cell shape, cytoskeletal tension, and RhoA regulate stem cell lineage commitment, Dev. Cell 6 (4) (2004) 483-495.

[38] G. Kocer, P. Jonkheijm, Guiding hMSC adhesion and differentiation on supported lipid bilayers, Adv. Healthc. Mater. 6 (3) (2017).

[39] D. Bocking, O. Wiltschka, J. Niinimaki, H. Shokry, R. Brenner, M. Linden, C. Sahlgren, Mesoporous silica nanoparticle-based substrates for cell directed delivery of Notch signalling modulators to control myoblast differentiation, Nanoscale 6 (3) (2014) 1490-1498.

[40] M. Yuasa, T. Yamada, T. Taniyama, T. Masaoka, W. Xuetao, T. Yoshii, M. Horie, H. Yasuda, T. Uemura, A. Okawa, S. Sotome, Dexamethasone enhances osteogenic differentiation of bone marrow- and muscle-derived stromal cells and augments ectopic bone formation induced by bone morphogenetic protein-2, PLoS One 10 (2) (2015) e0116462.

[41] Y. Wang W. Zhang. J. Zhang. W. Sun, R. Zhang H. Gu, Fabrication of a nove polymer-free nanostructured drug-eluting coating for cardiovascular stents, ACS Appl. Mater. Interf. 5 (20) (2013) 10337-10345. 